\title{
Silencing B7-HI enhances the anti-tumor effect of bladder cancer antigen-loaded dendritic cell vaccine in vitro
}

This article was published in the following Dove Press journal:

OncoTargets and Therapy

5 August 2014

Number of times this article has been viewed

\author{
Shuo Wang' \\ Yonghua Wang' \\ Jing Liu ${ }^{2}$ \\ Shixiu Shao' \\ Xianjun $\mathrm{Li}^{\prime}$ \\ Jiannan Gao' \\ Haitao Niu' \\ Xinsheng Wang' \\ 'Department of Urology, ${ }^{2}$ Department \\ of Pediatrics, The Affiliated Hospital of \\ Qingdao University, Qingdao, People's \\ Republic of China
}

Objective: The aim of this study was to examine whether short hairpin RNA (shRNA) expressing lentiviral particles targeting B7-H1 infection could result in B7-H1 knockdown on dendritic cells (DCs) and to investigate whether B7-H1 silencing could augment the immune function of DCs and further elicit a more potent anti-tumor immune effect against bladder cancer cells in vitro.

Methods: Monocyte-derived DCs, which were generated from peripheral blood mononuclear cells, were infected by a recombinant lentivirus containing shRNA sequence aimed at B7-H1. After that, the infected DCs were pulsed by tumor antigens and used to stimulate cytotoxic T lymphocytes-based anti-tumor effect in vitro.

Results: The lentivirus-mediated shRNA delivery method efficiently and effectively silenced B7-H1 in DCs. Furthermore, the B7-H1 silencing enhanced the stimulatory capacity and the secretion of interleukin-12, but down-regulated interleukin-10 secretion. And more importantly, the anti-tumor effect of bladder cancer antigen-loaded DC vaccine in vitro was also potentially augmented.

Conclusion: This study suggests that a combination of B7-H1 knockdown and target antigen delivery could augment anti-tumor effects in vitro, which potentially provides a novel strategy in the immunotherapy of bladder cancer.

Keywords: B7-H1, bladder cancer, dendritic cell, vaccine, immunotherapy

\section{Introduction}

Bladder cancer is the most common malignancy of the urinary tract, with high risks of recurrence and progression. ${ }^{1,2}$ Some studies have shown that bladder cancer patients have tumor associated immune dysfunction, which may contribute to the high recurrence rate and aggressive biological behavior. ${ }^{3}$ But the etiology of bladder cancerrelated immune dysfunction remains unclear.

Dendritic cells (DCs), which have been identified as the most potent antigen presenting cells (APCs), play a vital role in the initiation, programming, and regulation of tumor-specific immune responses. ${ }^{4,5}$ It has been shown that suppression of DCs' differentiation and function may contribute to immune dysfunction and cancer progression., ${ }^{46}$ B7-H1 (also known as PD-L1) is a recently discovered costimulatory molecule. It can inhibit immune responses by binding to its receptor PD-1 on the surface of T lymphocytes and consequently inducing antigen-specific T-cell apoptosis or anergy. ${ }^{7,8}$ Recently, some studies found that tumor microenvironment could up-regulate B7-H1 expression on DCs and then down-regulate T-cell immunity by suppressing DCs' function. ${ }^{9}$ Our previous study also demonstrated that B7-H1 expression was
Correspondence: Yonghua Wang Department of Urology, The Affiliated Hospital of Qingdao University, Qingdao 266000, People's Republic of China $\mathrm{Tel}+8618661805829$

Email doctorwangyonghua@I63.com 
significantly up-regulated on DCs from patients with bladder cancer. ${ }^{10}$ All these results implied that B7-H1 may be a potential target for improvement of DC function.

Therefore, in this study we constructed a recombinant lentivirus containing short hairpin RNA (shRNA) sequence aimed at B7-H1 and then infected DCs to investigate whether B7-H1 knockdown could augment the immune function of DCs and further elicit a more potent anti-tumor immune effect against bladder cancer cells. Here, we show that B7-H1 silencing can enhance the stimulatory capacity and Th1 type cytokine secretion of DCs. And more importantly, the antitumor effect of bladder cancer antigen-loaded DC vaccine in vitro is also potentially augmented.

\section{Materials and methods Materials}

Roswell Park Memorial Institute (RPMI) 1640 culture medium and fetal bovine serum (FBS) were purchased from HyClone (Logan, UT, USA). Recombinant human granulocytemacrophage colony stimulating factor (rhGM-CSF) and recombinant human interleukin-4 (rhIL-4) were purchased from PeproTech (Rocky Hill, CT, USA). Lipopolysaccharide (LPS) was purchased from Sigma-Aldrich Co (St Louis, MO, USA). Fluorescein isothiocyanate (FITC)-conjugated antiCD80, anti-CD83, and phycoerythrin (PE)-conjugated antiCD86, anti-HLA-DR were purchased from BioLegend (San Diego, CA, USA). Anti-B7-H1 polyclonal antibody was purchased from Santa Cruz Biotech (Dallas, TX, USA). Cell Counting Kit-8 (CCK-8) was purchased from Dojindo (Tokyo, Japan). IL-10 enzyme-linked immunosorbent assay (ELISA) kit, IL-12 ELISA kit, and IFN- $\gamma$ ELISA kit were purchased from R\&D Systems (Minneapolis, MN, USA). Lactate dehydrogenase (LDH) cytotoxicity assay kit was purchased from Beyotime (Nanjing, People's Republic of China).

\section{Cell line}

Human bladder cancer T24 cell line, derived from a patient with bladder cancer (HLA-A1+), was obtained from China Center for Type Culture Collection (CCTCC). Cells were cultured in RPMI 1640 (HyClone) with 10\% FBS (HyClone) and maintained at $37^{\circ} \mathrm{C}$ in a humidified atmosphere of $5 \% \mathrm{CO}_{2}$.

\section{Generation of monocyte-derived DCs}

Peripheral blood was obtained from healthy donors with informed consent, which was approved by the medical ethics committee at our institution. Before blood harvesting, the HLA-A genotype of each volunteer was examined using sequencing based typing (SBT) for screening of HLA-A1+, which matched that of the T24 cell line. Peripheral blood mononuclear cells (PBMCs) were isolated using FicollHypaque density gradient centrifugation and were incubated at $37^{\circ} \mathrm{C}$ and $5 \% \mathrm{CO}_{2}$ for 2 hours to allow plastic adherence. After the incubation, non-adherent cells were removed by washing these flasks gently. The adherent monocytes were then cultured in RPMI 1640 (HyClone) completed medium (containing 10\% FBS [HyClone]). The cultures were supplemented with 1,000 U/mL rhGM-CSF (PeproTech) and $1,000 \mathrm{U} / \mathrm{mL}$ rhIL-4 (PeproTech) for 5 days to generate immature dendritic cells (imDCs).

\section{Preparation of shRNA expressing lentiviral vector targeting $\mathrm{B} 7-\mathrm{HI}$}

The shRNA targeting human B7-H1 gene contained 21-nucleotide targeting sequence (5'-GGTTCCCAAGGACCTATATGT-3') which was separated by a loop from the reverse complement of the targeting sequence, a U6 terminator, and 2 cohesive ends for ligation into the pGLV-GFP vector, which was linearized by Age I and EcoR I. This recombinant vector was named pGLV-GFP-shB7-H1. The negative control vector (pGLV-GFP-NC) contained a nonsense sequence inserted in order to control any effects caused by transfection mechanisms. Both lentiviral vectors contained a sequence that expressed green fluorescent protein (GFP) which could be detected as a parameter reflecting transfection efficacy. We co-transfected the 293T-cell line with four-plasmid system (pVSV-G, pRev, pGag/Pol, pGLV-GFP-shB7-H1 or pGLVGFP-NC), which is essential for the production of lentiviral stocks. The culture supernatant was harvested 72 hours after transfection and filtered through $0.45 \mu \mathrm{m}$ filters. The final lentiviruses were named LV-GFP-shB7-H1 and LV-GFP-NC separately.

imDCs were collected on day 5 and divided into three groups: untreated group, LV-GFP-NC infected group (NC/DC), and LV-GFP-shB7-H1 infected group (shB7-H1/DC). The LV-GFP-shB7-H1 and LV-GFP-NC were added into the culture at a multiplicity of infection (MOI) of 30. After incubation for 24 hours, culture medium was replaced, supplemented with rhGM-CSF (PeproTech) $(1,000$ U/mL), rhIL-4 (PeproTech) (1,000 U/mL) and LPS (SigmaAldrich Co) $(1 \mu \mathrm{g} / \mathrm{mL})$ for another 2 days to generate mature DCs (mDCs). And lysates of T24 cells were added into the culture medium to obtain bladder cancer antigen-loaded DC vaccine. The lentiviral infection efficiency was measured by counting GFP-positive cells under fluorescence microscopy after infection for 72 hours. 


\section{The phenotype of DC vaccine}

DC phenotype was determined by flow cytometry using a series of antibodies (Abs) including FITC-conjugated anti-CD80, anti-CD83 and PE-conjugated anti-CD86, antiHLA-DR (BioLegend). The staining followed the same basic procedure. Briefly, imDCs and $\mathrm{mDCs}$ were collected and stained with $\mathrm{Abs}$ for 30 minutes at $4^{\circ} \mathrm{C}$. After washing twice with PBS, the cells were analyzed immediately using a FACSCalibur flow cytometry (Becton Dickinson, Franklin Lakes, NJ, USA).

\section{B7-HI expression on DC vaccine}

B7-H1 mRNA and protein expression on DCs were detected by reverse transcription-polymerase chain reaction (RT-PCR) and Western blot respectively. Total RNA was extracted from DCs by using TRIzol reagent (Thermo Fisher Scientific, Waltham, MA, USA). Two micrograms of total RNA was reverse transcribed and amplified with gene-specific primers using the RT-PCR System kit (TakaRa, Dalian, People's Republic of China). The primer sequences used were B7-H1 (forward, 5'-GCC GAC TAC AAG CGA ATT AC-3'; reverse, 5'-TCT CAGTGT GCT GGT CAC AT- $3^{\prime}, 234$ bp) and $\beta$-actin (forward, 5'-TCA TGG GTG TGA ACC ATG AGA A-3'; reverse, $5^{\prime}$-GGC ATG GAC TGT GGT CAT GAG-3, 146 bp). PCR conditions were 5 minutes at $95^{\circ} \mathrm{C}$, up to 38 cycles of 30 seconds denaturing at $95^{\circ} \mathrm{C}, 30$ seconds annealing at $58^{\circ} \mathrm{C}$, and 45 seconds extension at $72^{\circ} \mathrm{C}$ with final extension for 10 minutes at $72^{\circ} \mathrm{C}$. The PCR amplicons were analyzed by $1.5 \%$ agarose gel. Total proteins from DCs were separated on polyacrylamide gel, transferred onto nitrocellulose membranes and incubated with anti-B7-H1 (Santa Cruz Biotech) polyclonal antibody overnight at $4^{\circ} \mathrm{C}$. Membranes were then washed three times in washing buffer and incubated with anti-rabbit $\operatorname{IgG}$ conjugated to peroxidase for 1 hour at room temperature. Immunoreactivity was determined by chemiluminescence according to manufacturer's instructions.

\section{The stimulatory capacity and cytokines of DC vaccine}

The capacity to stimulate T-cell proliferation was measured by mixed lymphocyte reaction. Mature DCs of three groups were harvested and cocultured with autologous T-cells, isolated from PBMCs with immunomagnetic microbeads (Pan T Cell Isolation Kit; Miltenyi Biotec, Bergisch Gladbach, Germany) in 96-well plates respectively. Cultures were incubated for 4 days and then pulsed with $20 \mu \mathrm{L} /$ well of CCK-8 (Dojindo) for 4 hours. The absorbance of each well was measured with a microplate reader at $450 \mathrm{~nm}$ and each assay was repeated at least three times. Proliferation indices of T-cells were measured as stimulation index (SI) which was calculated as follows: $\mathrm{SI}=$ (experiment OD-blank OD)/ (negative control OD-blank OD). Meanwhile, the supernatants of cultures were harvested for cytokine assays. IL-10 and IL-12 secretions were detected by IL-10 ELISA kit and IL-12 ELISA kit (R\&D Systems) respectively according to the manufacturer's instructions.

\section{CTLs cytotoxicity assays}

Autologous T-cells were purified from PBMCs with immunomagnetic microbeads and cocultured with bladder cancer antigen-loaded DC vaccine in 96-well plates. After 1 week, a second identical stimulation was performed to generate tumor specific CTLs. T24 cells $\left(1 \times 10^{4} /\right.$ well $)$ were seeded in 96-well plates and used as targets for CTLs at an effector/target (E/T) ratio of 5:1, 10:1 and 20:1 $\left(5 \times 10^{4} /\right.$ well, $1 \times 10^{5} /$ well, and $2 \times 10^{5} /$ well CTLs) for 12 hours. LDH (Beyotime) release was then determined with LDH cytotoxicity assay kit and the percentage of specific lysis was calculated as: [(experimental release-spontaneous release $) /($ maximum release- spontaneous release $)] \times 100$. In addition, IFN- $\gamma$ production in the supernatants was also detected by IFN- $\gamma$ ELISA kit (R\&D Systems). IFN- $\gamma$ secretion capacity of CTLs was measured as IFN- $\gamma$ concentration per $5 \times 10^{4}$ CTLs, which excluded the influence of increased $\mathrm{E} / \mathrm{T}$ ratios and reflected the initiation effect of different $\mathrm{DC}$ vaccines.

\section{Statistical analysis}

Statistical analysis was performed using SPSS 17.0 (SPSS Inc., Chicago, IL, USA). Data were obtained and enumerated by Student's $t$-test. $P<0.05$ was regarded as statistically significant.

\section{Results}

\section{Lentiviral infection efficiency and the phenotype of $D C$ vaccine}

To evaluate the effect of B7-H1 silencing, we first designed shRNA expressing lentiviral particles targeting B7-H1 and infected bladder cancer antigen-loaded DC vaccine. We found that GFP-positive cells were identified in approximately $75.7 \% \pm 2.3 \%$ of all cells under fluorescence microscopy, which indicated remarkable infection efficiency (Figure 1). We further tested the phenotype of DC vaccine to exclude negative effects of lentiviral infection. As shown in Figure 2, all mDCs showed high expression of CD80, CD83, CD86, and HLA-DR in contrast to imDCs. In addition, the 

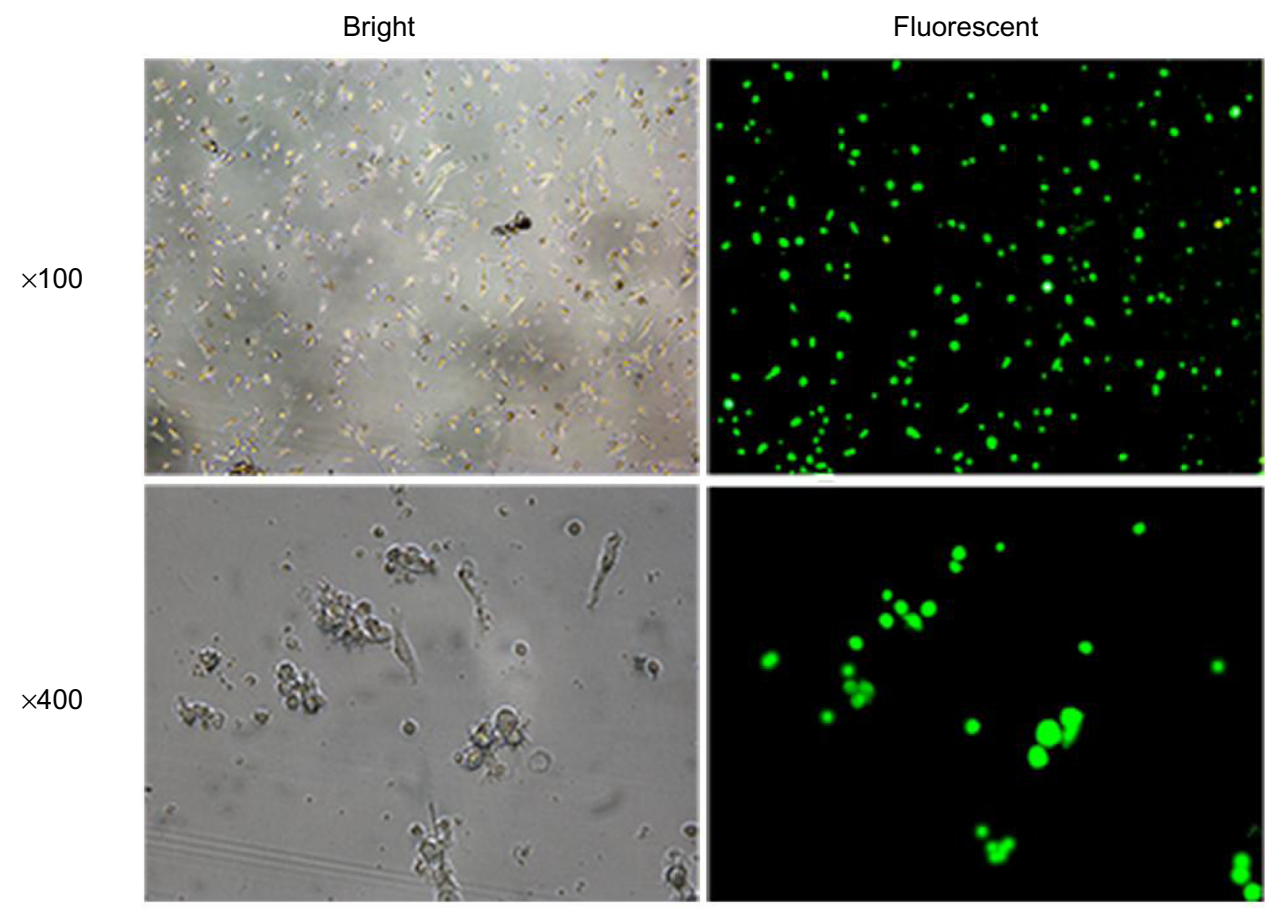

Figure I GFP expression under fluorescence microscopy after lentiviral infection.

Notes: DCs were infected with lentivirus at an MOI of 30 for 24 hours. GFP could be observed under fluorescence microscopy 72 hours after infection, which was ultimately identified in $75.7 \% \pm 2.3 \%$ of DCs. This GFP + percentage demonstrates a relatively high infection efficiency.

Abbreviations: MOI, multiplicity of infection; GFP, green fluorescent protein; DC, dendritic cell.

phenotype of DC vaccine among three groups exhibited no significant differences, indicating that virus infection did not affect the maturation and main phenotype of DC.

\section{ShRNA expressing lentiviral particles targeting B7-HI specially inhibits $\mathrm{B} 7-\mathrm{HI}$ expression on DC vaccine}

Consistent with previous studies, B7-H1 mRNA and protein expressions on DCs were up-regulated obviously after maturation. However, after infected with shRNA expressing lentiviral particles targeting B7-H1, B7-H1 expressions on DC vaccine were specially suppressed. Thus, we confirm that DCs infection with shRNA expressing lentiviral particles targeting B7-H1 can exert a potent knockdown effect on endogenous B7-H1 expression (Figure 3).

\section{Silencing of B7-HI enhances the immune function of $D C$ vaccine}

To investigate whether B7-H1 silencing could improve the immune function of DC vaccine, the capacity to stimulate T-cell proliferation was measured by mixed lymphocyte reaction and cytokine productions were analyzed by ELISA. We found that $\mathrm{DC}$ vaccine combined with $\mathrm{B} 7-\mathrm{H} 1$ silencing demonstrated a stronger capability to stimulate T-cell proliferation. Meanwhile, IL-12 secretion was increased and IL-10 secretion was decreased after B7-H1 silencing. Thus, our results indicate
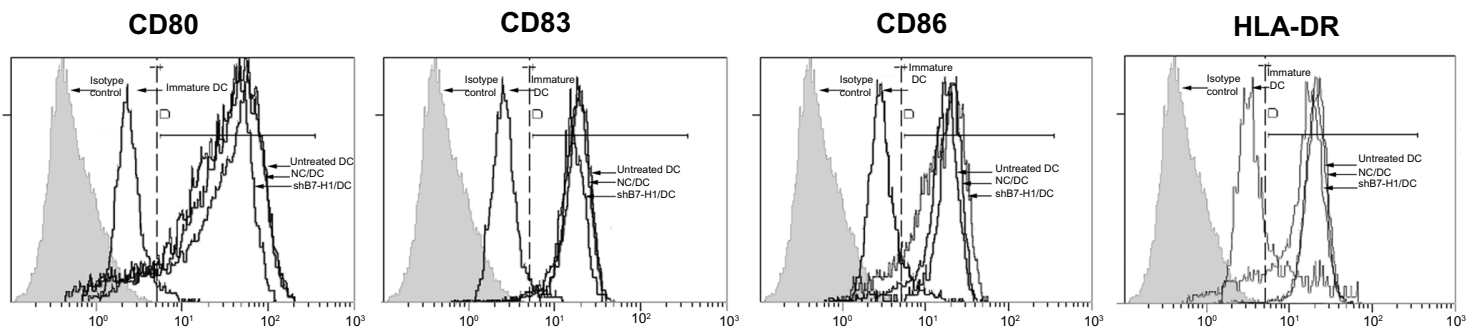

Figure 2 Expression of surface molecular markers on DC.

Notes: DCs were harvested and analyzed by flow cytometry using monoclonal antibodies. The results showed increased expressions of CD80, CD83, CD86, and HLA-DR, which is a typical phenotype of mature DC. Additionally, shB7-HI/DC, NC/DC, and untreated DC exhibited no significant differences, suggesting that lentiviral infection did not affect the maturation and main phenotype of DC. The data shown are representatives of three independent experiments.

Abbreviations: NC/DC, LV-GFP-NC infected DC; shB7-HI/DC, LV-GFP-shB7-HI infected DC; DC, dendritic cell. 
A

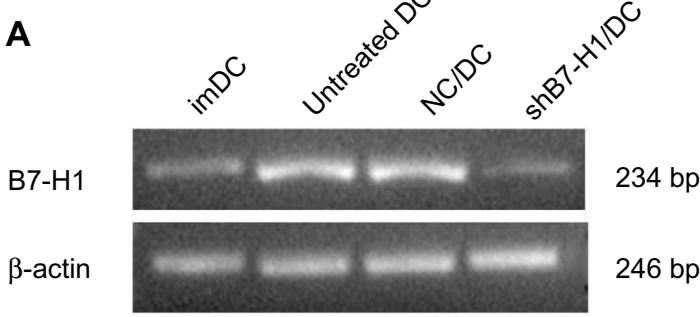

B

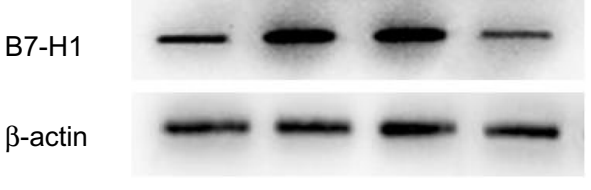

$50 \mathrm{KD}$

$43 \mathrm{KD}$

\section{C}

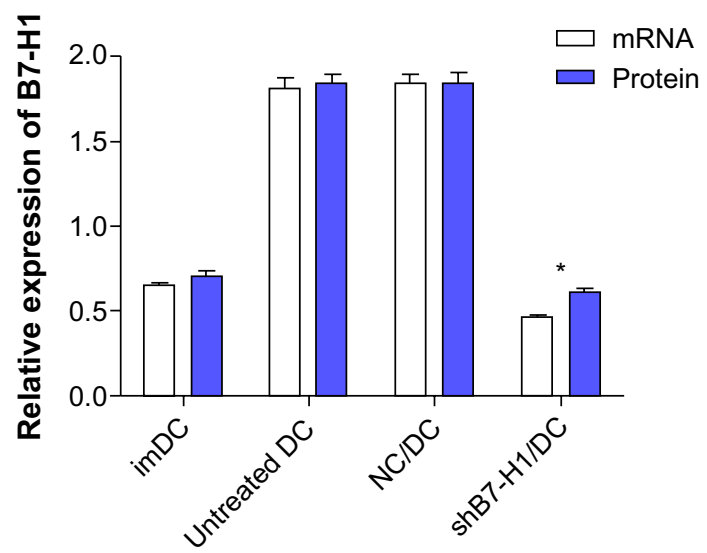

Figure 3 Detection of the B7-HI expression by RT-PCR and Western blot.

Notes: (A) RT-PCR analysis of the B7-HI mRNA. (B) Western blot analysis of the B7-HI protein. (C) Relative expression rate normalized to $\beta$-actin. B7-HI mRNA and protein were measured by RT-PCR and Western blot separately and were normalized to $\beta$-actin expression. Obvious up-regulation of B7-HI after maturation of DC was consistent with previous studies. As indicated, B7-HI in shB7-HI/DC was significantly suppressed at both mRNA and protein levels, showing that the shRNA-containing lentivirus could mediate a specific and potent knockdown effect on endogenous B7-HI expression. Results are representatives of three independent experiments. Data were shown as mean \pm standard deviation. $* P<0.05$ versus untreated $D C$.

Abbreviations: imDC, immature DC; NC/DC, LV-GFP-NC infected DC; shB7-HI/DC, LV-GFP-shB7-HI infected DC; DC, dendritic cell; RT-PCR, reverse transcriptionpolymerase chain reaction.

that B7-H1 silencing can enhance the stimulatory capacity and Th1 type cytokine secretion of DC vaccine (Figure 4).

\section{Silencing of B7-HI augments the anti-tumor effect of bladder cancer antigen-loaded DC vaccine in vitro}

For the beneficial effect of $\mathrm{B} 7-\mathrm{H} 1$ silencing on DC function, we further tested whether B7-H1 silencing could enhance the anti-tumor effect of bladder cancer antigen-loaded DC vaccine in vitro. We found that tumor specific CTLs induced by bladder cancer antigen-loaded DC vaccine combined with B7-H1 silencing expressed the highest level of IFN- $\gamma$ compared with the other groups. CTLs stimulated by antigenloaded DC secrete a modest level of IFN- $\gamma$. This indicates that $\mathrm{B} 7-\mathrm{H} 1$ silencing on DC promotes CTLs to produce more IFN- $\gamma$ (Figure 5A). LDH (Beyotime) release assay was also performed to test the cytolytic activity. As shown in Figure 5B, untreated DCs exhibited lowest anti-tumor activity which did not change at escalating E/T ratios. DCs pulsed by tumor antigen were observed to induce modest CTL activity against $\mathrm{T} 24$ cells and antigen-loaded DC infected by LV-GFPB7-H1 demonstrated the highest cytotoxic effect. This result
A

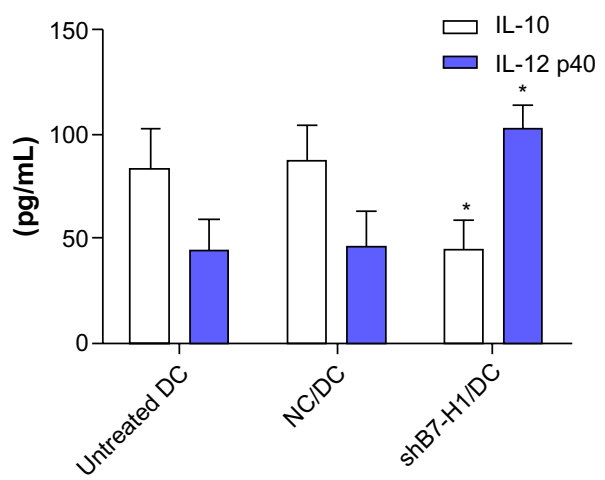

B

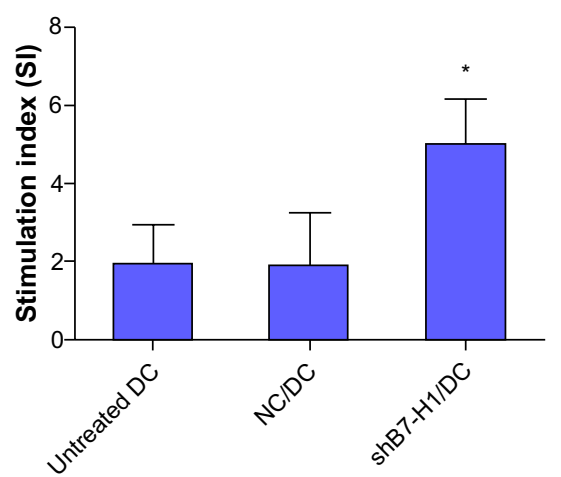

Figure $4 \mathrm{~B} 7-\mathrm{HI}$ silencing enhances ThI type cytokine secretion and stimulatory capacity of DCs.

Notes: (A) ELISA detection of IL- 10 and IL-12 production in DCs. (B) Stimulatory capacity in mixed lymphocyte reaction. The supernatants were collected and cytokines were analyzed by ELISA. B7-HI knockdown evidently increased IL-I 2 production and reduced IL- 10 secretion simultaneously. T-cells' proliferation was detected by CCK-8 kit (Dojindo, Tokyo, Japan). The result showed B7-HI-silenced DC elicited a stronger T-cell expansion in MLR. Assays were conducted in triplicate. Data were shown as mean \pm standard deviation. $* P<0.05$ versus untreated $D C$.

Abbreviations: IL, interleukin; NC/DC, LV-GFP-NC infected DC; shB7-HI/DC, LV-GFP-shB7-HI infected DC; DC, dendritic cell; MLR, mixed lymphocyte reaction. 
A

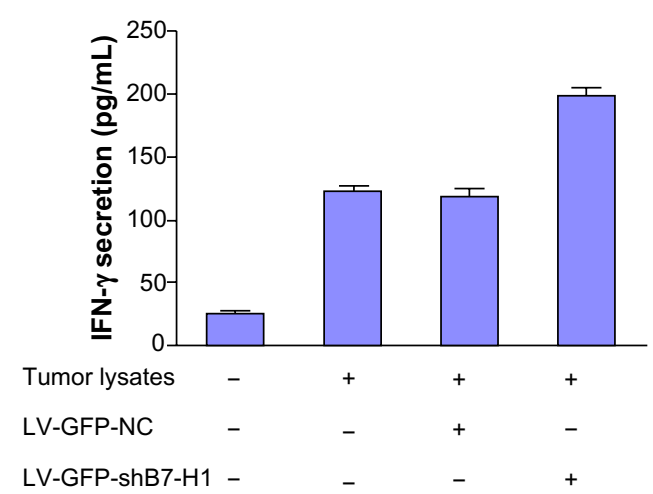

B

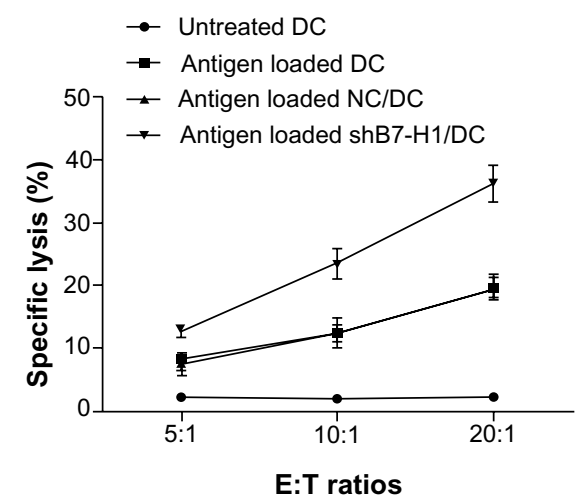

Figure 5 Anti-bladder cancer effect induced by DC vaccines.

Notes: (A) ELISA detection of IFN- $\gamma$ secretion (per $5 \times 10^{4}$ CTLs). (B) The cytotoxic effects of CTLs against bladder cancer T24 cell. CTLs primed by four types of DC vaccines were added into T24 cell cultures at an E:T ratio of 5:I, 10:I and 20:I. IFN- $\gamma$ secretion and cancer cell apoptosis rates were determined I2 hours later. The data showed that LV-GFP-shB7-HI infected DCs-stimulated CTLs expressed the highest level of IFN- $\gamma$ and had the highest cytotoxic effect among these four types of CTLs. Abbreviations: NC/DC, LV-GFP-NC infected DC; shB7-HI/DC, LV-GFP-shB7-HI infected DC; DC, dendritic cell; E:T, effector/target; CTLs, cytotoxic T lymphocytes.

indicated that DC pulsed by antigens could enhance the antitumor effect of CTLs and B7-H1 knockdown strategy could further improve this effect. Thus, we confirm that silencing of B7-H1 can augment the anti-tumor effect of bladder cancer antigen-loaded DC vaccine in vitro.

\section{Discussion}

T-cell mediated cytoimmunity is a main form of host antitumor immunity. Recognition and presentation of tumor antigen and activation of tumor specific T-cell is necessary for the start of tumor immune response. DCs, which are the most potent APCs, play an important role in the initiation, programming, and regulation of tumor immune responses. mDCs express high levels of MHC and costimulatory molecules. ${ }^{11}$ Stimulatory and inhibitory signals presented by DCs during antigen presentation are integrated by the T-cell and determine the final outcome of T-cell activation. ${ }^{11-13}$

B7-H1 is a recently discovered T-cell suppressive costimulatory molecule that is mainly expressed on the surface of activated monocytes and DCs. ${ }^{71}$ B7-H1 on DCs can provide co-inhibitory signals in the priming of cellular immune responses by binding to its ligands on T-cells, preventing autoimmunity and allowing the body to establish tolerance to self-antigens in normal physiology. ${ }^{15,16}$ It has been verified that inflammatory mediators in the tumor microenvironment can significantly up-regulate the expression of $\mathrm{B} 7-\mathrm{H} 1$ on tumor-associated DCs including those in tumor-draining lymph nodes and derived from ascites $\mathrm{CD} 14^{+}$monocytes, which subsequently contribute to the suppressive functions of DCs and facilitate tumor escape. ${ }^{9,17}$ Our previous study also demonstrated that B7-H1 expression was significantly up-regulated on DCs from patients with bladder cancer. ${ }^{10}$
It indicates that up-regulation of B7-H1 on DCs may be a new mechanism by which human cancers evade immunity.

The improved understanding of B7-H1 signaling in DC permits manipulation of this interaction and determines the therapeutic role of immunity in cancer. There are reports on the use of B7-H1 blockage to increase the efficacy of cancer immunotherapy. Importantly, in two recently published articles, Hobo et al efficiently delivered B7-H1 siRNA into DCs via lipid nanoparticles and demonstrated that these DCs could strongly enhance antigen-specific T-cell proliferation and cytokine production in allogeneic stem cell transplantation patients. ${ }^{18,19}$ However, the cytokine secretion of DC and the direct anti-tumor effect were not involved. Herein, we constructed shRNA expressing lentiviral particles targeting B7-H1 and then infected DCs to investigate whether B7-H1 knockdown could augment the immune function of DCs and further elicit a more potent anti-tumor immune effect against bladder cancer cells. We found that DC vaccine combined with B7-H1 silencing demonstrated a stronger capability to stimulate T-cell proliferation. Meanwhile, IL-12 secretion was increased and IL-10 secretion was decreased after B7-H1 silencing. DCs can secrete many cytokines to regulate immune responses. Some cytokines can augment Th1 type responses which promote T-cell mediated cytoimmunity, while others can induce immunotolerance. It is well known that IL-12 is pivotal for enhancing Th1 type immunity and anti-tumor responses, while IL-10 facilitates Th2 type immunity which is undesirable and tumor-promoting. Our data suggest that the expression of B7-H1 seems to affect the endogenous signaling in DC. Though the exact mechanisms need to be further elucidated, it is certain that targeting the B7-H1 immune checkpoint can enhance the immune function of DC vaccine 
by altering the secretion profile of DC. Furthermore, tumor specific CTLs induced by bladder cancer antigen-loaded DC vaccine combined with B7-H1 silencing demonstrated a stronger cytotoxicity against T2 4 cells and IFN- $\gamma$ production of tumor specific CTLs was also enhanced. Thus, we confirm that silencing of B7-H1 can augment the anti-tumor effect of bladder cancer antigen-loaded DC vaccine in vitro.

Bladder cancer has been characterized as an immunogenic cancer that contains large amounts of tumor-infiltrating lymphocytes and is sensitive to immunotherapy with bacillus Calmette-Guérin. ${ }^{20,21}$ Thus, bladder cancer is considered as one of the most responsive human malignancies to immunotherapy, including DC vaccine. The present DC vaccine strategies focus on the presentation of tumor antigens. For example, DCs can be loaded with proteins derived from tumors, synthetic peptides, RNA/DNA encoding specific tumor antigen or other forms. ${ }^{22-24}$ Nevertheless, the negative regulation of B7-H1 is ignored. We speculate that the infused DC vaccines enter the tumor microenvironment where B7-H1 expression could be up-regulated and this phenotype alteration may lead to functional impairment. This mechanism may be involved, at least in part, in the dysfunction of DC vaccine in clinical trials. Our study suggests that targeting B7-H1 may become a potential strategy for improving the anti-tumor effect of DC vaccine. Therefore, we believe the $\mathrm{B} 7-\mathrm{H} 1$ silencing strategy is crucial to improve the clinical potential of current DC-based immunotherapy. Moreover, B7-H1 shRNA modified DC vaccine may be a promising adjuvant therapeutic modality for bladder cancer and should be used in combination with current therapeutics for improvements in survival.

In conclusion, we have demonstrated that B7-H1 silencing can enhance the immune function of DC and augment the anti-tumor effect of bladder cancer antigen-loaded DC vaccine in vitro. Currently, B7-H1/PD-1 antibodies have a very broad potential and trials in many tumor types are being initiated. We suggest that targeting B7-H1 combined with DC vaccine may also become a highly promising strategy in immunotherapy and further in vivo studies are warranted.

\section{Acknowledgment}

This work was supported by the National Natural Science Foundation of China (No 81101932).

\section{Disclosure}

The authors indicate no conflicts of interest.

\section{References}

1. Ploeg M, Aben KK, Kiemeney LA. The present and future burden of urinary bladder cancer in the world. World J Urol. 2009;27(3):289-293.
2. Babjuk M, Burger M, Zigeuner R, et al. EAU guidelines on non-muscleinvasive urothelial carcinoma of the bladder: update 2013. Eur Urol. 2013;64(4):639-653.

3. Loskog A, Ninalga C, Paul-Wetterberg G, de la Torre M, Malmstrom PU, Totterman TH. Human bladder carcinoma is dominated by T-regulatory cells and Th1 inhibitory cytokines. J Urol. 2007;177(1):353-358.

4. Banchereau J, Steinman RM. Dendritic cells and the control of immunity. Nature. 1998;392(6673):245-252.

5. Steinman RM, Banchereau J. Taking dendritic cells into medicine. Nature. 2007;449(7161):419-26.

6. Ma Y, Shurin GV, Peiyuan Z, Shurin MR. Dendritic cells in the cancer microenvironment. J Cancer. 2013;4(1):36-44.

7. Dong H, Zhu G, Tamada K, Chen L. B7-H1, a third member of the B7 family, co-stimulates T-cell proliferation and interleukin-10 secretion. Nat Med. 1999;5(12):1365-1369.

8. Dong H, Strome SE, Salomao DR, et al. Tumor-associated B7-H1 promotes T-cell apoptosis: a potential mechanism of immune evasion. Nat Med. 2002;8(8):793-800.

9. Curiel TJ, Wei S, Dong H, et al. Blockade of B7-H1 improves myeloid dendritic cell-mediated antitumor immunity. Nat Med. 2003;9(5): $562-567$.

10. Ji C, Wang Y, Yu Q, Liu J, Liu Y, Cui J. Expression and significance of B7-H1 in peripheral blood dendritic cells from patients with bladder cancer. Chinese-German J Clin Oncol. 2013;12(6):290-292.

11. Banchereau J, Briere F, Caux C, et al. Immunobiology of dendritic cells. Annu Rev Immunol. 2000;18:767-811.

12. Greenwald RJ, Freeman GJ, Sharpe AH. The B7 family revisited. Annu Rev Immunol. 2005;23:515-548.

13. Zang X, Allison JP. The B7 family and cancer therapy: costimulation and coinhibition. Clin Cancer Res. 2007;13(18 Pt 1):5271-5279.

14. Freeman GJ, Long AJ, Iwai Y, et al. Engagement of the PD-1 immunoinhibitory receptor by a novel B7 family member leads to negative regulation of lymphocyte activation. J Exp Med. 2000;192(7): 1027-1034.

15. Dong H, Zhu G, Tamada K, Flies DB, van Deursen JM, Chen L. B7-H1 determines accumulation and deletion of intrahepatic CD8(+) T lymphocytes. Immunity. 2004;20(3):327-336.

16. Hori J, Wang M, Miyashita M, et al. B7-H1-induced apoptosis as a mechanism of immune privilege of corneal allografts. J Immunol. 2006;177(9):5928-5935.

17. Yang W, Song Y, Lu YL, Sun JZ, Wang HW. Increased expression of PD-1 and its ligand PD-L1 correlates with impaired cell-mediated immunity in high-risk HPV-related cervical intraepithelial neoplasia. Immunology. 2013;139(4):513-522.

18. Hobo W, Maas F, Adisty N, et al. siRNA silencing of PD-L1 and PD-L2 on dendritic cells augments expansion and function of minor histocompatibility antigen-specific CD8+ T-cells. Blood. 2010;116(22): 4501-4511.

19. Hobo W, Novobrantseva TI, Fredrix H, et al. Improving dendritic cell vaccine immunogenicity by silencing PD-1 ligands using siRNA-lipid nanoparticles combined with antigen mRNA electroporation. Cancer Immunol Immunother. 2013;62(2):285-297.

20. Tsujihashi H, Matsuda H, Uejima S, Akiyama T, Kurita T. Immunoresponse of tissue infiltrating lymphocytes in bladder tumors. J Urol. 1989;141(6):1467-1470.

21. Patard JJ, Saint F, Velotti F, Abbou CC, Chopin DK. Immune response following intravesical bacillus Calmette-Guerin instillations in superficial bladder cancer: a review. Urol Res. 1998;26(3):155-159.

22. Bhargava A, Mishra D, Banerjee S, Mishra PK. Dendritic cell engineering for tumor immunotherapy: from biology to clinical translation. Immunotherapy. 2012;4(7):703-718.

23. Kirkwood JM, Butterfield LH, Tarhini AA, Zarour H, Kalinski P, Ferrone S. Immunotherapy of cancer in 2012. CA Cancer J Clin. 2012;62(5):309-335.

24. Kalinski P, Muthuswamy R, Urban J. Dendritic cells in cancer immunotherapy: vaccines and combination immunotherapies. Expert Rev Vaccines. 2013;12(3):285-295. 


\section{Publish your work in this journal}

OncoTargets and Therapy is an international, peer-reviewed, open access journal focusing on the pathological basis of all cancers, potential targets for therapy and treatment protocols employed to improve the management of cancer patients. The journal also focuses on the impact of management programs and new therapeutic agents and protocols on

patient perspectives such as quality of life, adherence and satisfaction. The manuscript management system is completely online and includes a very quick and fair peer-review system, which is all easy to use. Visit http://www.dovepress.com/testimonials.php to read real quotes from published authors.

Submit your manuscript here: http://www.dovepress.com/oncotargets-and-therapy-journal 